\title{
At the origins of the Trojan Horse Method
}

\author{
Marcello Lattuada
}

Dipartimento di Fisica ed Astronomia, Via S. Sofia 64, 95123 Catania, Italy

Istituto Nazionale di Fisica Nucleare- LNS, Via S. Sofia 62, 95123 Catania, Italy

\begin{abstract}
During the seventies and eighties a long experimental research program on the quasi-free reactions at low energy was carried out by a small group of nuclear physicists, where Claudio Spitaleri was one of the main protagonists. Nowadays, a posteriori, the results of these studies can be considered an essential step preparatory to the application of the Trojan Horse Method (THM) in Nuclear Astrophysics.
\end{abstract}

Everything began in the seventies, with the publication on Nuclear Physics A of a paper reporting the results of an experiment carried out by a group of Japanese researchers on the three alpha-particles produced in the ${ }^{3} \mathrm{He}+{ }^{9} \mathrm{Be}$ reaction at incident energy of $4 \mathrm{MeV}$ [1]. The coincidence detection of two of the outgoing alpha particles showed that, in addition to the usual sequential decay through states of ${ }^{8} \mathrm{Be}$, another mechanism contributed to the spectra, giving place to a bump centered at the minimum momentum of the undetected alpha. Angular correlations and energy sharing spectra showed that the shape of this bump was consistent with a contribution of the ${ }^{5} \mathrm{He}\left({ }^{3} \mathrm{He}, \alpha\right){ }^{4} \mathrm{He}$ virtual reaction occurring in the frame of a quasi-free process. In this process the undetected alpha cluster $\alpha_{\mathrm{s}}$ acts as spectator of the process and keeps the momentum it owns at the time of the interaction, as schematized in fig. 1.

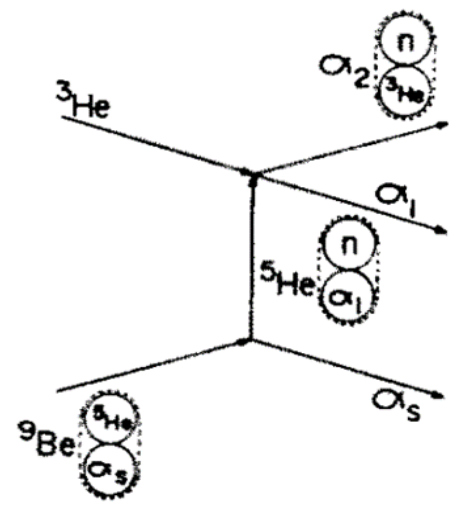

Fig. 1. Schematic picture of the ${ }^{3} \mathrm{He}+{ }^{9} \mathrm{Be}$ quasi-free reaction mechanism (from [1]).

In this representation the reaction proceeds through two independent steps corresponding to two virtual processes: the virtual decay of one of the particles in the entrance channel $\left({ }^{9} \mathrm{Be}\right.$ into ${ }^{4} \mathrm{He}$ and ${ }^{5} \mathrm{He}$ in this case) and the virtual reaction of one of the decay products $\left({ }^{5} \mathrm{He}\right)$ with the other participant $\left({ }^{3} \mathrm{He}\right)$. No one of the two processes conserves the energy separately but of course they conserve the energy on the whole. 
The dominant s-wave motion of the ${ }^{4} \mathrm{He}$ and ${ }^{5} \mathrm{He}$ clusters in ${ }^{9} \mathrm{Be}$ produces a maximum in the ${ }^{9} \mathrm{Be}\left({ }^{3} \mathrm{He}, \alpha \alpha\right)^{4} \mathrm{He}$ spectra in correspondence of the minimum spectator momentum, according to the Impulse Approximation (IA) predictions, which for the triple differential cross section gives a simple formula:

$$
\frac{d^{3} \sigma}{d E_{1} d \Omega_{1} d \Omega_{2}} \approx K F \boldsymbol{\Phi}^{2}\left(\boldsymbol{p}_{s}\right) \frac{d \sigma}{d \Omega}
$$

Here $\Phi^{2}\left(p_{s}\right)$ is the squared momentum distribution of the spectator particle, that in the Plane Wave IA (PWIA) is simply given by the Fourier transform of the cluster wave function, $\mathrm{d} \sigma / \mathrm{d} \Omega$ is the half-off-energy-shell cross section of the virtual two body reaction and $\mathrm{KF}$ is a factor proportional to the phase space. Actually the use of the IA requires a high momentum transfer and the Q-value of the three body reaction guarantees that this requirement was satisfied, indeed. On the other hand the use of the PWIA instead of the more realistic Distorted Wave IA (DWIA) approach was justified since it has been shown that in many cases the PWIA satisfactorily approximates the DWIA in the region close to the minimum spectator momentum. Of course the PWIA analysis cannot provide any information on the absolute value of the cross section.
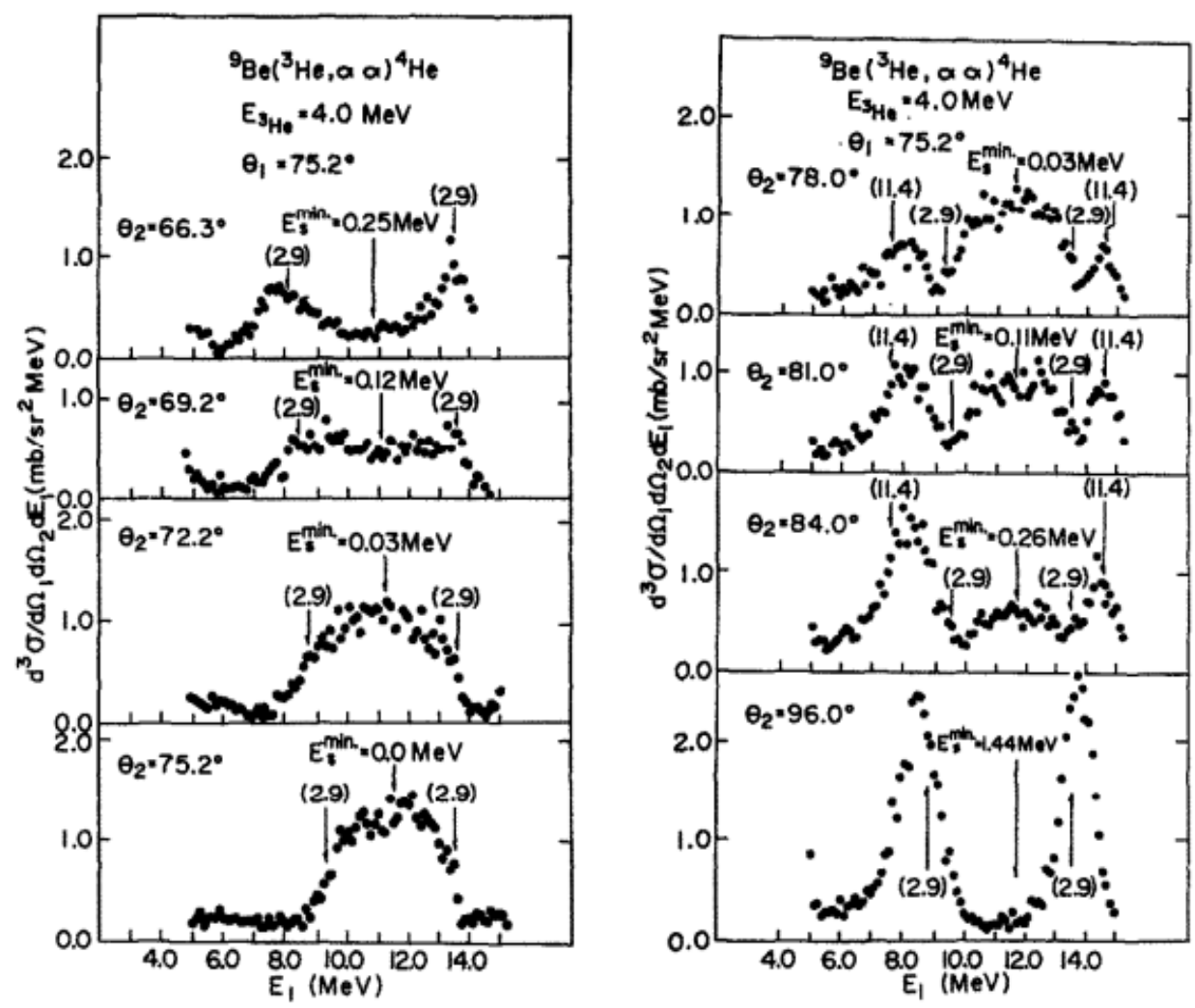

Fig. 2. Alpha-alpha coincidence spectra projected on the energy of one of the detected alpha-particles from the ${ }^{9} \mathrm{Be}\left({ }^{3} \mathrm{He}, 2 \alpha\right){ }^{4} \mathrm{He}$ reaction for various angle pairs (from [1])

The question arose whether such contribution was present also at lower energy and/or in other similar systems. Actually the occurrence of a quasi-free scattering like $(p, p \alpha)$ or $(\alpha$, 
$2 \alpha$ ) in the interaction of light nuclei had already been found in earlier years for some other light systems even at energy of some tens of $\mathrm{MeV}$, but on the other hand very few were the systems showing the contribution of quasi-free reactions at so low energy.

At that time a small single stage Van de Graaff, with a maximum voltage of about $3 \mathrm{MV}$, was operating at the Catania Physics Department. So the experiment was repeated with this accelerator at the sub-barrier energy of $2.8 \mathrm{MeV}$ with a set of detectors covering the phase space regions where the minimum ${ }^{4} \mathrm{He}$ spectator momentum assumes different values, with the purpose of following the evolution of the differential cross section [2]. In this experiment and in experiments performed later at different incident energies [3] the appearance of a quasi-free reaction contribution well described by Eq. 1 was again confirmed and coherent shapes of the momentum distribution were extracted from all the coincidence spectra.

In addition the pole character of the mechanism was tested with a later experiment, applying the Treiman-Yang criterion to the ${ }^{3} \mathrm{He}+{ }^{9} \mathrm{Be}$ reaction [4]. The isotropy of the Treiman-Yang distribution, even if it is a necessary but not sufficient condition, represented a further support to the independence of the two poles.

According to a standard procedure, the quasi-free data were analyzed in two steps. First a phase space region where the relative energy and center of mass angles of the two interacting particles are appreciably constant was selected to deduce the experimental momentum distribution using the Eq.1. Then, dividing the three body cross section by the experimental momentum distribution and by the kinematical factor, it was possible to deduce, again from Eq.1, the virtual two body cross section as a function of angle and energy. Of course no comparison with the free cross section could be done in the case of the ${ }^{5} \mathrm{He}\left({ }^{3} \mathrm{He}, \alpha\right){ }^{4} \mathrm{He}$ reaction, due to the unbound nature of ${ }^{5} \mathrm{He}$.

Other light systems were experimentally studied in the following years (see for instance [58] and references therein), possessing in the entrance channel projectile or target with a marked ground state cluster structure, like ${ }^{6} \mathrm{Li}$ and ${ }^{2} \mathrm{H}$ and indication of a quasi-free mechanism was found in many cases. The case of the ${ }^{6} \mathrm{Li}\left({ }^{6} \mathrm{Li}, 2 \alpha\right)^{4} \mathrm{He}$ reaction [9] was particularly interesting since the $\alpha$-d momentum distribution in ${ }^{6} \mathrm{Li}$ could be measured within the same experiment by selecting alternatively the events corresponding to the quasifree mechanism with the spectator either in the target or in the projectile. The coincidence cross section, divided by the proper kinematical factor, for events corresponding to the two quasi-free mechanisms, showed similar resonant behavior peaked at the same energy as reported in Fig. 3.

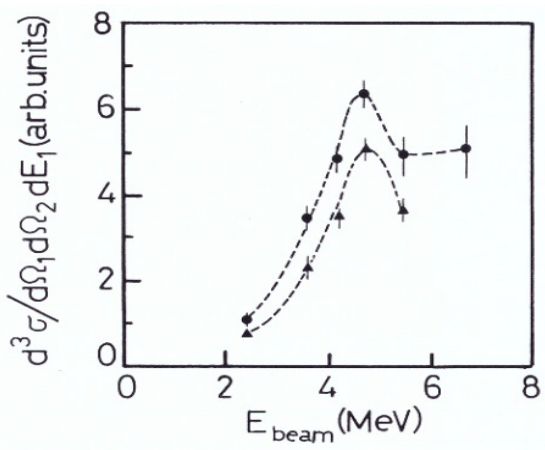

Fig. 3. Excitation function of the ${ }^{6} \mathrm{Li}\left({ }^{6} \mathrm{Li}, 2 \alpha\right)^{4} \mathrm{He}$ reactions at around zero of the spectator $\alpha$-particle momentum, either in the target (points) or in the projectile (triangles). The cross sections are divided by the proper kinematical factor. Figure from [9]. 
But the most clear indication came from two experiments which allowed the extraction of the two body ${ }^{1} \mathrm{H}\left({ }^{6} \mathrm{Li}, \alpha\right){ }^{3} \mathrm{He}$ and ${ }^{1} \mathrm{H}\left({ }^{7} \mathrm{Li}, \alpha\right){ }^{4} \mathrm{He}$ cross sections down to sub-barrier energies, from the three body ${ }^{2} \mathrm{H}\left({ }^{6} \mathrm{Li}, \alpha^{3} \mathrm{He}\right) \mathrm{n}$ and ${ }^{2} \mathrm{H}\left({ }^{7} \mathrm{Li}, 2 \alpha\right) \mathrm{n}$ reactions respectively. The agreement of the THM excitation functions with the free reaction data was impressive, probably also due to the absence of Coulomb interaction between the spectator neutron and the participants. The resonances corresponding to excitation of known states of ${ }^{7} \mathrm{Be}$ (at 7.2 $\mathrm{MeV}$ ) [10] and ${ }^{8} \mathrm{Be}$ (at 19.9 and $22.2 \mathrm{MeV}$ ) [11] in the ${ }^{1} \mathrm{H}\left({ }^{6} \mathrm{Li}, \alpha\right){ }^{3} \mathrm{He}$ and ${ }^{1} \mathrm{H}\left({ }^{7} \mathrm{Li}, \alpha\right){ }^{4} \mathrm{He}$ reactions respectively were fairly reproduced by the THM data. The comparison between free and quasi-free data is shown in fig. 4 where the fits to the free reaction data are reported as continuous curves, whilst the points represent the virtual reaction cross sections.
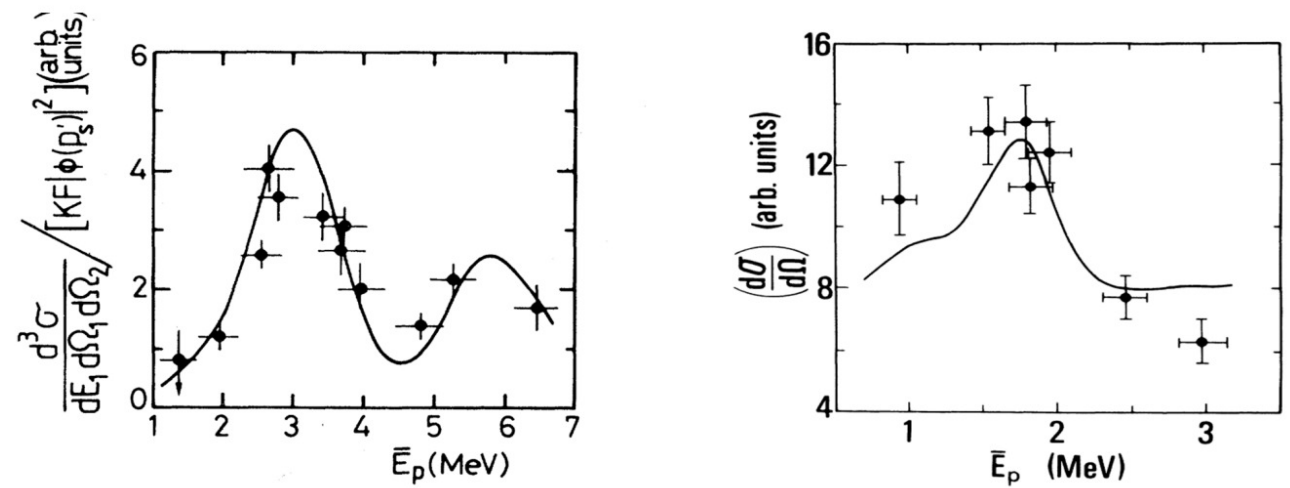

Fig. 4. Two body ${ }^{1} \mathrm{H}\left({ }^{7} \mathrm{Li}, \alpha\right)^{4} \mathrm{He}$ (left, from [10]) and ${ }^{1} \mathrm{H}\left({ }^{6} \mathrm{Li}, \alpha\right)^{3} \mathrm{He}$ (right, from [11]) excitation functions extracted from the ${ }^{2} \mathrm{H}\left({ }^{7} \mathrm{Li}, 2 \alpha\right) \mathrm{n}$ and ${ }^{2} \mathrm{H}\left({ }^{6} \mathrm{Li}, \alpha{ }^{3} \mathrm{He}\right) \mathrm{n}$ three body reactions respectively.

It was a clear, experimental confirmation that one can indirectly measure a two body reaction cross section even at very low interaction energy by choosing a suitable three body reaction at energy above the Coulomb barrier and selecting appropriate kinematical regions where the quasi-free contribution is favored. Thanks to the high projectile energy, the entrance channel relative energy can exceed the Coulomb barrier. Once the barrier has been overcome, the virtual reaction can occur even at very low relative energies, resulting from the energy balance where the deuteron binding energy and the $n-p$ relative motion inside the deuteron are taken into account. Then the first important implication of the success of the method was the possibility of an indirect measurement of astrophysical reaction cross sections whose low interaction energy often makes it difficult, and sometimes prohibitive, a direct experimental approach. Moreover another interesting perspective on the side of nuclear physics is that the quasi-free mechanism can be regarded as a unique tool for the study of reactions with an unbound nucleus in the entrance channel.

The possibility of using the deuteron as virtual source of low energy protons or, more interesting, neutrons opened new opportunities not only in nuclear astrophysics but also in nuclear physics. For this reason and also for its low binding energy and its well-known momentum distribution, the deuteron can be considered as the ideal Trojan Horse nucleus. Claudio Spitaleri firmly believed in the use of quasi-free reactions in nuclear astrophysics [12] and devoted his efforts to the involvement of a quite large number of researchers in international collaborations working on this topic. The result is that important improvements have been introduced in these years, from the point of view of the THM formalism as well as from the point of view of the use of more sophisticated experimental set-ups. Nowadays the THM has an important role as a tool for the indirect study of nuclear astrophysical processes, together with the Coulomb dissociation and the ANC method [13]. Around Claudio Spitaleri, a group of young researchers grew in Catania attracted by the 
simplicity and wide perspectives of this powerful tool and by the enthusiasm for the studies of nuclear astrophysics transmitted by him.

It is difficult to mention the many collaborators and colleagues who were involved with Claudio Spitaleri in these studies, without forgetting anybody. So big is the net of collaborations developed on this subject. Nevertheless a special mention is deserved by some of them who, for different, and sometimes sad, reasons could not attend this NPA8 special session, but have contributed in different ways and times to the study of the quasifree reaction mechanism and its relevance in the development of the THM.

Mimmo Vinciguerra must be remembered for bringing into the group his previous experience in the field of quasi-free scattering. Gerhard Baur gave an enlightening contribution with the introduction of the original concept of the Trojan Horse. Herman Wolter contributed with his competence to the development of the THM formalism. The continuous encouragement of Claus Rolfs along with his decisive push for the foundation of the Santa Tecla European Summer School on Experimental Nuclear Astrophysics cannot be forgotten. A deep gratitude has to be expressed to Djuro Miljanic for sharing his wide knowledge of the light nuclei interaction and for thirty-five years of collaboration and friendship.

\section{References}

1. J. Kasagi, T. Nakagawa, N. Sekine, T.Tohei, H. Ueno, Nucl. Phys. A 239, 233 (1975)

2. N. Arena, D. Vinciguerra, F. Riggi, C. Spitaleri, Lett. N. Cimento 17, 231 (1976)

3. M. Lattuada, F. Riggi, C. Spitaleri, D. Vinciguerra, D. Miljanic, M. Zadro, Yao Jinzhang, Nucl. Phys. A 458, 493 (1986)

4. P.G. Fallica, M. Lattuada, F. Riggi, C. Spitaleri, C.M. Sutera, D. Vinciguerra, Phys. Rev. C 24, 1394 (1981)

5. M. Lattuada, F. Riggi, S. Barbarino, C. Spitaleri, D. Vinciguerra, Lett. N. Cimento 20, 193 (1977)

6. M. Lattuada, F. Riggi, C. Spitaleri, Vinciguerra, Lett. N. Cimento 33, 433 (1982)

7. M. Lattuada, F. Riggi, C. Spitaleri, D. Vinciguerra, C.M. Sutera, Il N. Cimento A 71, 429 (1982]

8. S. Blagus, C, Blyth, G. Calvi, O. Karban, M. Lattuada, D. Miljanic, F. Riggi. C. Spitaleri, Z. Phys. A - Atomic Nuclei 337, 297 (1990)

9. M. Lattuada, F, Riggi, D. Vinciguerra, C. Spitaleri, D. Miljanic, Z. Phys. A - Atomic Nuclei 328, 497 (1987)

10. M. Zadro, D. Miljanic, C. Spitaleri, G. Calvi, M. Lattuada, F. Riggi, Phys. Rev. C 40, 181 (1989)

11. G. Calvi, M. Lattuada, D. Miljanic, F. Riggi, C. Spitaleri, D. Vinciguerra, Phys. Rev. C, 41, 1848 (1990)

12. C. Spitaleri, Proc. Fifth Hadronic Physics Winter Seminar, Folgaria-TN, Italy (Ed. World Scientific, Singapore) (1990)

13. R. Tribble C. A. Bertulani, M. La Cognata, A. M. Mukhamedzhanov C. Spitaleri, Rep. Prog. Phys. 77, 106901(2014) 
